\title{
MyRio based Automated Gear Transmission for Manual Gear Cars
}

\author{
Josephine Selle J, K Gokul Vasan, V Devendran, K Jeganadhan
}

\begin{abstract}
Technology plays a vital role in developing new ideas in the automobile industry. Currently optimization techniques and artificial intelligence have enhanced lot of features in the cars of higher end in terms of safety. Though these cars perform better authentically, they are less affordable and cannot be implemented for low cost cars. This project is an attempt to implement "automatic gear transmission (AGT) for manual cars, without altering the basics design of the automobile. The purpose of AGT is to avoid major accidents that occur due to nervousness and when the speed goes over control during the accidents such mis happenings can be eradicated when AGT is applied in particularly three modes such as normal track, hill climbing and reverse mode of operations. The success of this project would be greatly benefited for the smart India process.
\end{abstract}

Keywords-AGT, MyRio, LabVIEW stimulation, gear shift logics, motors, sensor.

\section{INTRODUCTION}

In the current century, technology plays a greater role within the world. Particularly in the automobile industry, the factors come with optimizing technology and their updated version. Years back, the optimization of the automobile industry exists the car equipped with automatic gear shifting technology, due to the advancement its cost also rapidly increased effectively when compared to manual gear transmission cars. The cost difference between the two cars almost became high in automatic gear transmission cars and avoid accidents.

To eradicate both the problems, we came with an innovative solution as Automated Gear Transmission (AGT). It can be implemented in the existing car without any alteration. From that the innovation over speeding can be controlled and avoid accidents in roadways. Then accidents happened by two factors, they are sudden deviation of the vehicle in the same or opposite lane and lagging of concentration due to nervousness. This can be completely eradicated from this invention. The aspects of the invention having three different modes, listed as normal track mode, hill climbing mode and reverse modes of operation. This invention would be the emphasis and optimization of the manual gear transmission systemcars.

Revised Manuscript Received on December 05, 2019.

* Correspondence Author

Josephine Selle J*, Dept. of ECE, Kalasalingam Academy of Research and Education, Krishnankoil, Tamil Nadu, India. Email: josephine@klu.ac.in

K Gokul Vasan, Dept. of ECE, Kalasalingam Academy of Research and Education, Krishnankoil, Tamil Nadu, India.

V Devendran, Dept. of ECE, Kalasalingam Academy of Research and Education, Krishnankoil, Tamil Nadu, India.

K Jeganadhan, Dept. of ECE, Kalasalingam Academy of Research and Education, Krishnankoil, Tamil Nadu, India.

\section{RELATEDWORKS}

By referring to some related publications a few limitations are pointed out such as; in [1] the hydraulic pressure control is achieved by improving shift quality. For this limitation, within the system discussed in this research, the power train effects and hydraulic pressure changes during at shift process this research has not only provided a intuitive understanding of shift processes. [2] A tool shift analyzer is used to calibrate the driving strategy offline. for this method limitation is, using a current transmission calibration consequent use of modern offline tools and processes enables FEV to be an efficient partner for today's and future transmission application projects. [3] It introduce a system with solenoid and cylinder type materials. This paper puts forward automatic shift $s$ the results prove that the institution can control the joystick accurately and make the transmission shift periodically, circularly and efficiently System platform. [4] This system consists of a permanent magnet type dc motor operates an electromagnetic clutch- type gear. [5] Gear ratio calculation in manual gear cars by analyzing the vehicle dynamics the limitation is so this method allows, different gearbox design module to be compared with others respectively, as accurately as possible, rather than the establishment of nominal values of considered exploitation parameters. [6] Driving simplification through four-stage automatic gearbox the limitation is a significant decrease of gearbox cost, enhancement of reliability with multiples, clutch operation with low costs, enhancement of driver's attention concentration will improve with the appropriate $n$ road conditions and enables the improvement of the trafficsafety.

[7] Investigated the hybrid electric vehicle (HEV) by using gear steps and motor operations the limitation is from the reverse simulation, it would be found that the fuel economy was improved as the number of gear step increased with the appropriate speed conditions, since the engine thermally efficiency increased and power electronics system loss decreased. [8] An adaptive control method to improve shift quality the limitation is about testing results stability of the adaptive strategy and it shows the feasibility. [9] Automotive gearbox implementation using methodological clutching sequence in this paper the limitation presents asystematic approach in designing for a gearbox structure for the purpose of obtaining an optimal shifting sequence with efficient manner. [10] Cars acceleration controlling by brake comfort model this paper is the limitation fact is that it can find a brake comfortable system with car-following model for longitudinal acceleration, whereas most former car- 


\section{MyRio based Automated Gear Transmission for Manual Gear Cars}

[8] following models only consider verticalvibration [21-24].

\section{WORKINGMETHODOLOGY}

The concept behind this invention is to convert mechanical operation into an electrical pulse, which operates an electronic controller. The speedometer detects the speed of the car and converts to digital representation from the sensor, this signal is fed as input to the lab view simulator and second input as a clutch pedal. The three different modes are taken into consideration, they are normal track mode, hill climbing mode and reverse modes ofoperation.

These modes fully operate on the speedometer and rpm value. Each of the modes has different programs and it works on the speedometer and rpm performance. In normal track mode the speed of the car could be at the certain speed of $15 \mathrm{mph}$, from the speed performance and rpm value it will be shifted as $2^{\text {nd }}$ gear. Similarly, for hill climbing scenario, the car speed performance and rpm value could be changed, as a function of different gear shifting. The block diagram of automatic gear transmission for manual gear cars is shown in the fig.1. Lab view simulator performs the input operation, depends upon the input value operation the gear shifting will be operated. During initialize start time of the car, only one mode will be enabled the remaining two will be a disabledcondition.

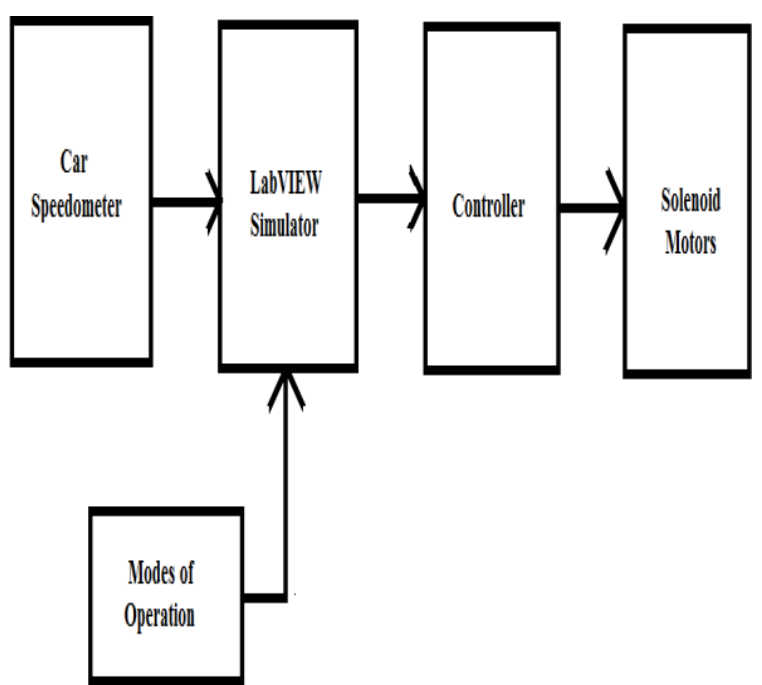

Fig. 1. Block Diagram

For example, if we press the reverse mode, normal track, and hill climbing mode will be turned disabled, only reverse mode program executed. The speed in mph will be programmed for individuals' modes. All the operationwouldbeexecutedandenablinginthesimulationitself, after that the simulator interfacing with the controller module, to enable the efficiency of the program based upon the input simulation and perform the long lasting of the device is high compared to other electronics devices. The controller fetches the signal from the simulator, depends on the fetching signal the solenoid motor works.

The operation of solenoid motor used to shift the gear forward and backward movements. Gear shifting operation enables with the input speedometer value, required simulator performance, and clutch pedal. Initially, the solenoid motor requires a control signal and a source of energy for their functioning. To trigger the motor, the control signal from the controller the gear shifting operation of a car could be performed. Though this car speed could be control at maximum speed while driving and to avoid accidents. The general workflow is shown in fig. 2

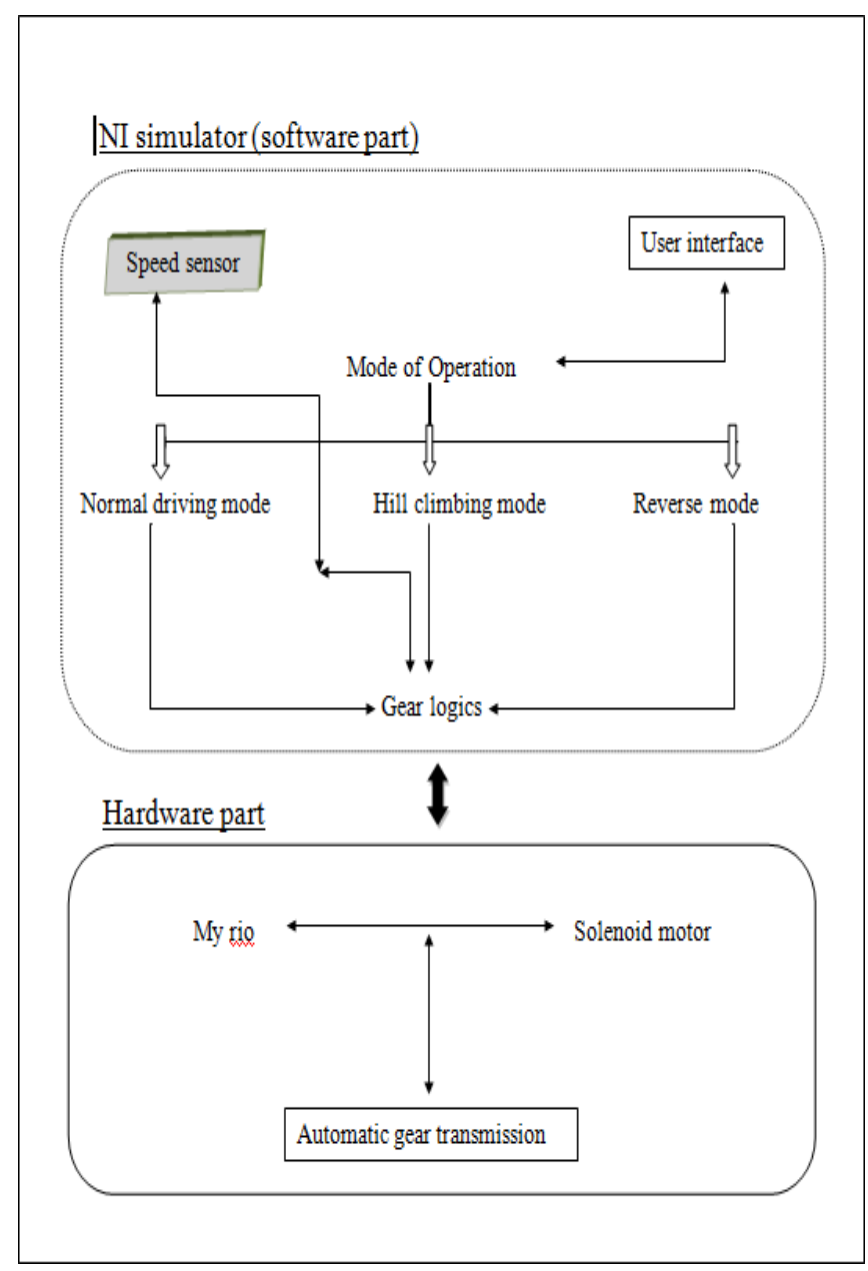

Fig. 2. Flow Chart

In the above flow chart, it consists of both software and hardware i.e., NI simulator and hardware part like solenoid motors. In this software part, we use three modes of operations which relate to speed sensor output. And these modes are the user interface and for different modes, we apply different logics through NI simulator.

Next, this software part output relates to the hardware part which includes MyRio kit and solenoid motor. Myrio kit performs as a controller, processor and the operating system. So, the software part output relates tothemyriokitandthiskithelpstoworksondifferent logic which is applied for modes of operations then the output of it relates to solenoid motor, it helps to shift the gear automatically depending upon clutch and speed of the car.

\section{COMPONENTS}




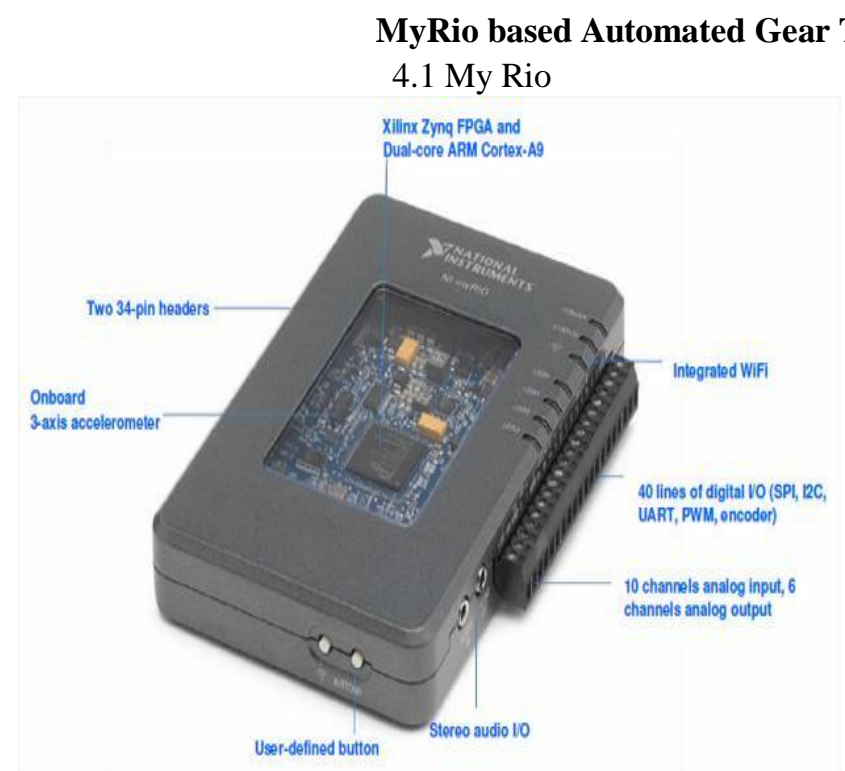

Fig. 3. Specifications of Myrio Kit

- Chip with Xilinx zynq system

- Analog Input with 10Channels

- Analog Output with 6Channels

- Through $3.5 \mathrm{~mm}$ Audio Jacks with analog input and analog outputsystem

- Digital I/O Lines as 40 pins; Wireless systemEnabled

- Onboard Accelerometer, LEDs, and Push Button alsoavailable

- Power source requirement as $6 \mathrm{~V}$ to $16 \mathrm{~V}, 14 \mathrm{~W}$

- Compatible with National Instrument MyRIO Module; Powered byLabVIEW

\section{A. Solenoid motors}

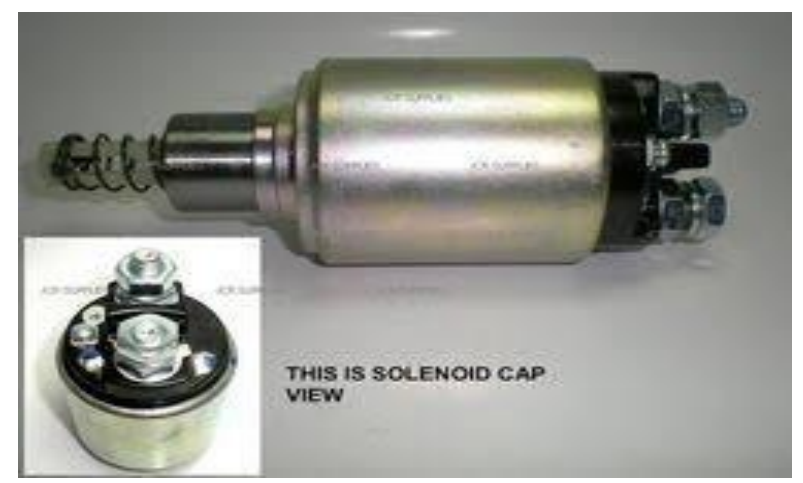

Fig. 4. Solenoid motor

As shown in the fig. 4. the solenoid motor is a type of coil of wire used as an electromagnetic purpose. It is having the basic principle as conversion of electric energy into mechanical energy [11-14]. The device which produce a magnetic field from electric current and uses this magnetic field to enable the linear motion. A solenoid motor is the part of an automobile which enables switches a large electric current to the motor, in response action to the small control current, and whichin turn used to sets the engine in motion. solenoid engines are generally in automobile engines run on electrical energy instead of chemical energy. It also equipped to any device that converts electric energy to mechanical energy using a solenoid device motor. Common real time applications of solenoids are to power a switch, like a starter in an automobile, or a valve, such as in a sprinkler system and all automobile equipment.

A solenoid motor is a coil of wire in a screw shape wrapped around a piston, made up of iron. For all electromagnets, a magnetic field is created when an electric current pass through the wire. Electromagnets having an advantage compared to permanent magnets in that they can be switched on and off by the applications of the electric current, which is used to makes them useful as switches and valves and allows them to be entirely automated. As in all magnets, the magnetic field of an activated solenoid has positive and negative poles that will attract or repel material sensitive to magnets. In a solenoid motor, the electromagnetic field causes the piston to either move backward or forward, which is how motion is created by a solenoid coil.

\section{B. Speedometer Sensor}

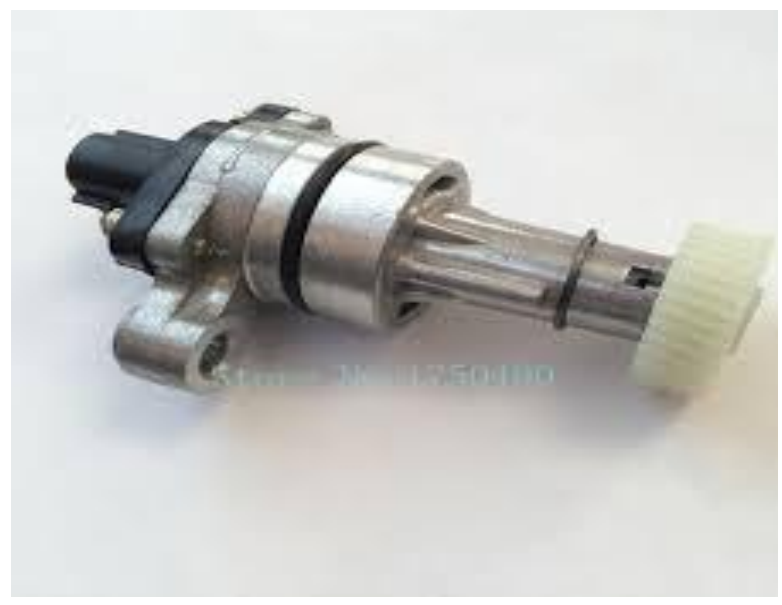

Fig. 5. Speedometer sensor

In fig. 5. a speedometer sensor is shown. With damage in a vehicle, this sensor can cause a list of problems. However, the most common symptoms associated with a damage engine speed sensor: The car's transmission in a car enables into overdrive delay and it will never go into that top gear of the gear system. There are generally twospeed sensors in an automobile that work in simultaneously to provide accurate transmission data pass to the vehicle's power train control system [15]. They are known as the input shaft speed (ISS) sensor. Purpose of this sensor is used to monitor the speed of the transmission' input shaft and provide output to the display. A proximity sensor is a sensor able to detect the presence of objects with nearby without any physical contact. It used to emit an electromagnetic field and looks for continuous changes intheelectromagnetic field $[16,17]$. A receiver detects the reflected waves and calculates the distance from your vehicle to the object. 


\section{RESULTS AND DISCUSSIONS}

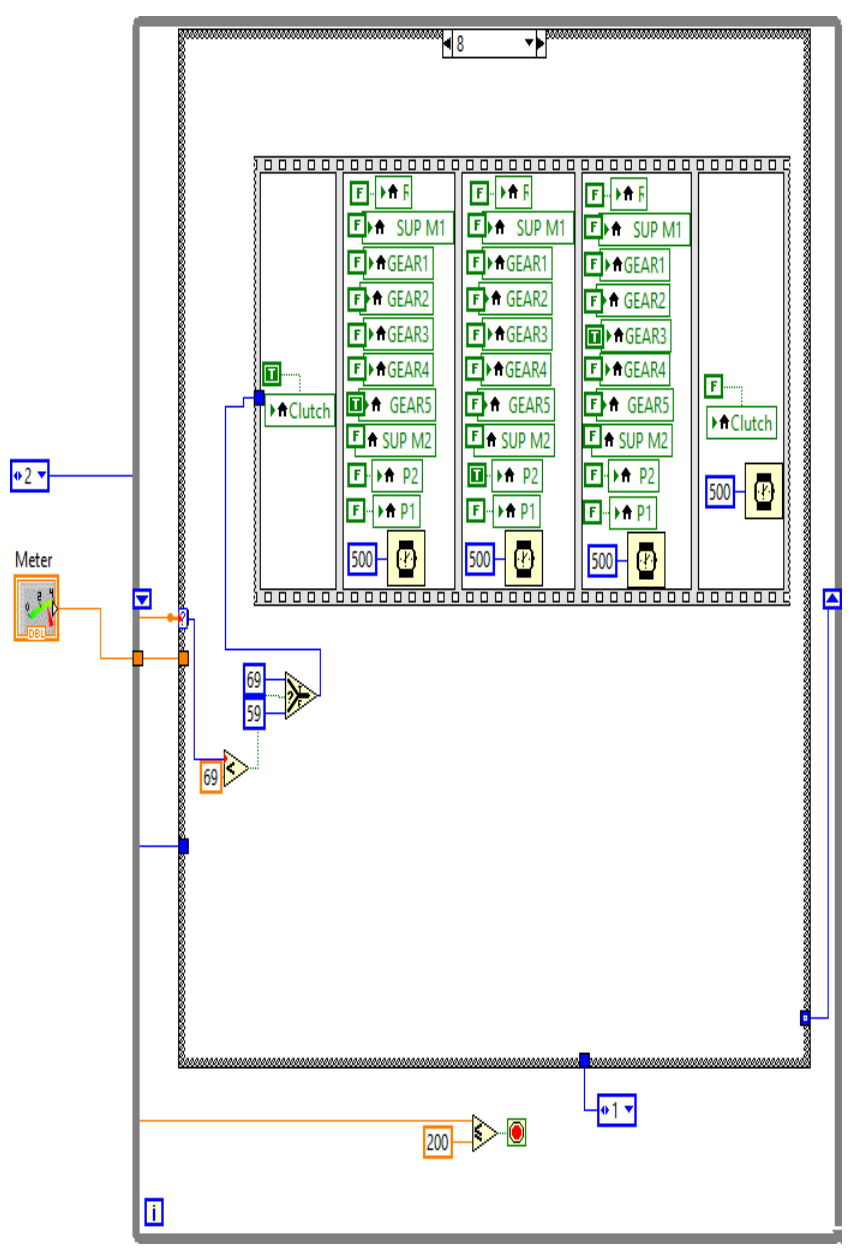

Fig.6.Block Diagram of Ni Stimulator

In $\mathrm{Ni}$ lab-view simulator all the required tools are available [18-20], so just we select the required tools and easily verify the result. Therefore, here we showed our output in Ni simulator, in this simulator, there are two parts. One is block diagram and another one is a front panel; it is nothing but user interface application. Here in the front panel, we use one analog speedometer, because for shifting gear it needs the speed of a car. And for representing motor movements we use eleven led lights. Wehave already explained, that is here we shift the gears through solenoid motor which is in to and from motion. Therefore, for representing these motors here, LED like a prototype. For easy understanding above we mentioned the user interface application diagram fig.7. in this diagram we fix the name for each led's, i.e., gear1, gear2, gear3, SUPM1, P1etc. here SUPM1, SUPM2, and P1, P2 is used to pull the gear for long distance. $\mathrm{R}$ is neutral gear. for clutch, we use the separate long pullingmotor.

The block diagram which is shown in fig. 6 , here we describe the diagram by creating a unique architecture for gear shifting. Here we use three case structure, first case structure is for speedometer logic, second case structure is for clutch automation and the last one is for gear automation. And, for every gear shifting, we maintain time using a timer.

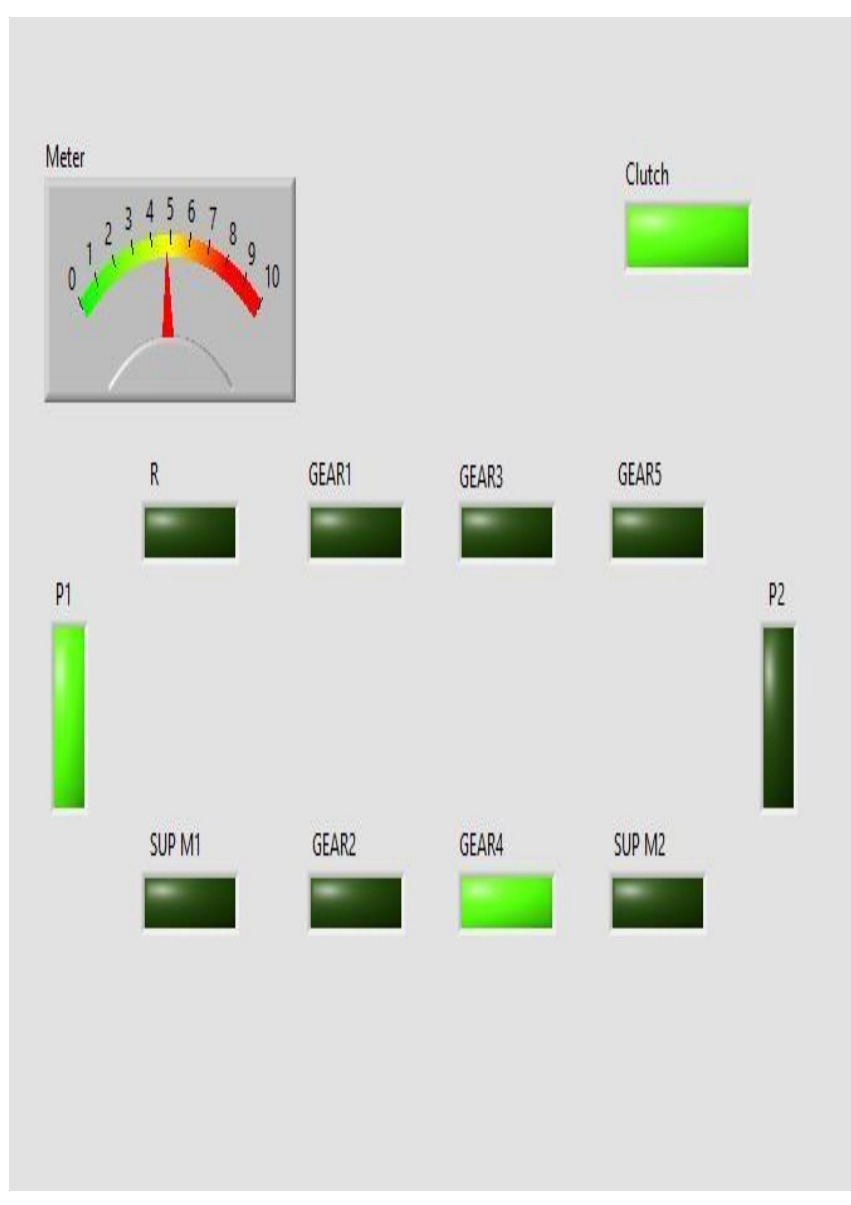

Fig. 7. User Interface Application Output

\section{CONCLUSION}

The proposed system talks about the AGT that is implemented in the manual gear transmission system cars without altering its internal structures. The performance of manual gear cars remains like the automatic gear transmission system cars.

In current automobile market by comparing both manual and automatic gear transmission cars, the cost efficiency is quite high in automatic gear transmission system cars which also have the provision to avoid accidents in roadways with rapid speed control. In order to overcome the issues, came with a solution namely "automatic gear transmission for manual gear cars". The proposed work emphasizes the optimization of the manual gear transmission system cars and it would step further towards "Smart India". Invention with such a low cost would attract customers not only within India, but it also attracts customers from various developingnations.

\section{REFERENCES}

1. Kuo, Kei-Lin. "Simulation and Analysis of the Shift Process for an Automatic Transmission." World Academy of Science, Engineering and Technology 52 (2011):341-347. 


\section{MyRio based Automated Gear Transmission for Manual Gear Cars}

2. Kirschstein, Stefan, Filipe Remelhe, and Bernd Stolze. "Efficient transmission application by using modern offline tools." Proceedings of 8th CTI International Symposium of Innovative Automotive Transmission, Berlin.2009.

3. Cheng, Tianyou, et al. "Research and development of automatic gear shifting system for automobile manual transmission." 2016 3rd International Conference on Materials Engineering, Manufacturing Technology and Control. Atlantis Press, 2016.

4. Ohmae, Tsutomu, et al. "Methods and characteristics of automatic gear change control of electric vehicle." Electrical Engineering in Japan 99.6 (1979): 27-37.

5. Bera, Piotr. "A design method of selecting gear ratios in manual transmissions of modern passenger cars." Mechanism and Machine Theory 132 (2019): 133-153.

6. Rassokha, Vladimir, and Vladimir Isaychev. "New design of the automobile automatic gearbox providing driving simplification and driver fatigue decrease." Transportation Research Procedia 20 (2017):544-549.

7. Kim, Hyunhwa, et al. "Influence of number of gear step on engine and motor operation characteristics for parallel HEV." Ecological Vehicles and Renewable Energies (EVER), 2018 Thirteenth International Conference on. IEEE, 2018.

8. Zhenkun, Dai, Dong Peng, and Guo Wei. "Adaptive strategy for the automatic transmission shifting improvement." Control And DecisionConference(CCDC), 2017 29th Chinese. IEEE, 2017.

9. Tamada, Sireesha, Debraj Bhattacharjee, andPranab

10. K. Dan. "Modeling and Formulation of Optimal Clutching Sequence in Automatic Transmission." 2018 IEEE Vehicle Power and Propulsion Conference (VPPC). IEEE,2018.

11. Wu, Zhaohui, Yanfei Liu, and Gang Pan. "A smart car control model for brake comfort based on car following." IEEE Transactions on Intelligent Transportation Systems 10.1 (2009):42-46.

12. Sun Wentao, Chen Huiyan, Wu Chunci. Shifting process adaptive control strategy with electronic automatic transmission[J]. Journal of Mechanical Engineering,2009,45(1):293-299.

13. Lu Xi, Guo Wei, Wang Shuhan, Xu Xiangyang. Application of shift control parameter adaption strategy for automatic transmission[J]. Transactions of the Chinese Society of Agricultural Engineering, 2015,31(12):83-91.

14. Zhang Tao, Tao Gang, Chen Huiyan. Control strategy for the torque phase in up-shift process of an electrically controlled automatic transmission[J]. Automotive Engineering,2013,35(2):173-178.

15. Li Xing hua, Ye Wei, Liu Zhao, Huang Zongyi. Dynamic Analysis of Automatic Transmission during Shifting[J]. Journal of Tongji University (Natural Science),2003,31(5):576-580.

16. Dai Zhenkun, Xu Xiangyang, Liu Yanfang, Wang Shuhan. Closedloop Slip Control of Clutches in Hydraulic Automatic Transmission. Automotive Engineering, 2012, 34(8):718-722.

17. Dai Zhenkun, Liu Yanfang, Xu Xiangyang, Wang Shuhan. Drive line modeling and shift characteristic simulation of automatic transmission power train system[J]. Journal of Beijing University of Aeronautics and Astronautics,2012,38(8):1027-1031.

18. Yan Yiquan, Song Jian, Li Liang. Multi-section Optimization Shift Control Method of Dry Dual Clutch Transmission[J]. Transactions of the Chinese Society for Agricultural Machinery,2014,45(5):30 36 .

19. Zhang Jinle, Ma Biao, Zhang Yingfeng, Li Yanhe. Shift Dynamics and Control of Dual Clutch Transmissions[J]. Transactions of the Chinese Society for Agricultural Machinery,2010,41(5):6-11.

20. Hebbale, Kumaraswamy, V.; Kao, C.-K.: Adaptive Control of Shifts in Automatic Transmissions. Proceedings of ASME International Mechanical Engineering Congress and Exposition, San Francisco, CA, 1995 .

21. Aström, K.J.; Wittenmark, B.: Adaptive Control. Addison-Wesley Publishing Company, 1989.

22. Ramadhan, R. M., Sudiarso, A., \&Mahardika, M. (2019, January). Design and Simulation of Auto-Shifting System in Manual Transmission Gearbox for Electric Race Car with Fuzzy Logic Controller on Matlab-Simulink. In Journal of Physics: Conference Series (Vol. 1150, No. 1, p. 012050). IOP Publishing.

23. Carlson, D. F., Fürsterling, A., Vesterled, L., Skovby, M., Pedersen, S. S., Melvad, C., \&Rysgaard, S. (2019). An affordable and portable autonomous surface vehicle with obstacle avoidance for coastal

\author{
ocean monitoring. Hardwarex, 5, e00059.
}

24. Rafiq, A. A., \& Yusuf, M. (2018, October). Implementation of Digital Image Processing Using NI myRIO and Arduino Mega 2560 as Controller On Rover Bogie Robot. In 2018 International Conference on Applied Science and Technology (iCAST) (pp. 210215). IEEE.

25. Chadry, R., \&Suryani, A. I. (2018). Embedded System Using Field Programmable Gate Array (FPGA) myRIO and LabVIEW Programming to Obtain Data Patern Emission of Car Engine Combustion Categories. INTERNATIONAL JOURNAL ON INFORMATICS VISUALIZATION (JOIV), 2(2), 56-62.

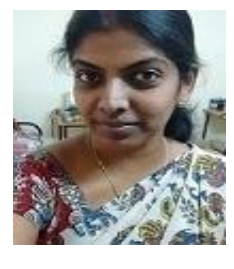

\section{AUTHORS PROFILE}

Josephine Selle Jcompleted her under-graduation in Electronics and Communication Engineering from Karunya University, Coimbatore and completed her postgraduation in Communication Systems from Anna University Affiliated College. Her research areas are Image Processing, Artificial Intelligence for medical images.

V Devendran, K Gokul Vasan and K Jeganadhan are undergraduate students from Kalasalingam Academy of Research and Education, Krishnankoil. There area of interests areprogramming with LabView and MyRio. 